\title{
Infant Affective Responses to Mother's Still Face at 6 Months Differentially Predict Externalizing and Internalizing Behaviors at 18 Months
}

\author{
Ginger A. Moore \\ Duke University
}

\author{
Jeffrey F. Cohn and Susan B. Campbell \\ University of Pittsburgh
}

\begin{abstract}
This study investigated (a) stability and change in infant affective responses to the still-face interaction, (b) whether maternal depression affected infant responses, and (c) whether responses to the still-face interaction predicted toddler problem behaviors. Infants (63 girls and 66 boys) of European American mothers (67 depressed and 62 nondepressed) were observed in the still-face interaction at 2, 4, and 6 months. Affect and gaze were coded on a 1-s time base. There were stable individual differences in gazing away and in rates of negative affect. Developmental change occurred only for gazing away, which increased. At 18 months, infants who failed to smile at 6 months in the still-face interaction showed more externalizing-type behaviors than did other toddlers. Infants who failed to cry at 6 months showed fewer internalizing-type behaviors. Mothers' current depressive symptoms and infants' earlier responses to the still-face interaction made independent, comparable contributions to problem behaviors at 18 months.
\end{abstract}

The still-face interaction, in which parents are instructed to become unresponsive to their infants after a period of normal social play, was designed to assess infants' affective responses to a change in contingent social interaction (Tronick, Als, Adamson, Wise, \& Brazelton, 1978). A large body of work has described the normative responses of infants between the ages of 3 and 9 months to the still-face interaction (e.g., Cohn \& Tronick, 1983; Field, Vega-Lahr, Scafidi, \& Goldstein, 1986; Murray \& Trevarthen, 1985; Shapiro, Fagen, Prigot, Carroll, \& Shalan, 1998; Toda \& Fogel, 1993; Tronick et al., 1978; Weinberg \& Tronick, 1994, 1996). Normative responses include brief smiles, negative facial expressions, crying, looking away, directed hand movements, and self-soothing behaviors such as thumb sucking. Researchers have interpreted these responses as infants' attempts to regain their parents' attention by smiling, distress at the lack of responsiveness, and attempts to regulate negative emotion by looking away or using self-soothing techniques, all of which suggest an emerging social-emotional competence.

Variation from normative responses to the still-face interaction may reflect important developmental factors and may mark later differences in children's social-emotional competence. However, developmental and individual differences in infant affective responses to the still-face interaction and the predictive validity of

Ginger A. Moore, Department of Psychology, Duke University; Jeffrey F. Cohn and Susan B. Campbell, Department of Psychology, University of Pittsburgh.

This research was supported by Grants MH40867 and MH56604 from the National Institute of Mental Health. Portions of this research were presented at the biennial conference of the International Society for Infant Studies, April 1998, Atlanta, Georgia.

Correspondence concerning this article should be addressed to Ginger A. Moore, Department of Psychology, Duke University, Durham, North Carolina 27708. Electronic mail may be sent to gmoore@duke.edu. these responses have not been researched widely. In prior work, variation has been considered in terms of age-related change (Gusella, Muir, \& Tronick, 1988; Shapiro et al., 1998; Toda \& Fogel, 1993), the impact of maternal depression (Field, 1984), and individual differences (Shapiro et al., 1998; Toda \& Fogel, 1993). Data regarding the predictive validity of infant affective responses to the still-face interaction are limited to relations with 12-month attachment behaviors. In the current study, we examined developmental change and individual differences in affective responses to the still-face interaction in a large sample of healthy infants, including a group of infants whose mothers were diagnosed with depression during the postpartum period. To examine predictive validity, at 18 months we compared the problem behaviors of infants whose earlier responses to the still-face interaction were normative with the problem behaviors of infants who had not shown the normative responses.

\section{Developmental Change}

With the exception of an increase in self-regulatory behaviors (e.g., gazing away, directed hand movements, thumb sucking), no age-related changes in normative responses to the still-face interaction from 3 to 6 months have been found (Gusella et al., 1988; Shapiro et al., 1998; Toda \& Fogel, 1993). Given that socialemotional development undergoes rapid change during this age period and that infants' expressions of positive emotion and responsiveness during mother-infant interactions increase (Cohn \& Tronick, 1987; Kaye \& Fogel, 1980), it may be that increases in self-regulatory behaviors are responsible for maintaining consistent rates of affect in the still-face interaction.

However, there are also methodological explanations to consider. First, sample sizes in previous studies have been relatively small, and there may have been insufficient power to detect developmental change. Second, developmental change in positive 
and negative responses may occur earlier than the 3-month time point examined in prior studies. Third, prior research has examined developmental change in terms of the rates of positive and negative responses. Another method is to look for developmental change in the number of infants who show the normative positive and negative responses (smiling and crying, respectively). In the current study, we observed infant positive and negative responses to the still-face interaction and gazing away as a self-regulatory behavior (a) in a much larger sample of healthy, full-term infants than has been studied previously, (b) beginning at an earlier age, and (c) across shorter developmental increments than were used in other studies. In addition to examining developmental change in the average rates of positive and negative responses, we examined change in the number of infants who smiled and the number of infants who cried across 2 to 6 months.

\section{Maternal Depression}

Although age-related changes in the rates of infant positive and negative affective responses to the still-face interaction have not been reported, another line of research has found differences in the responses of infants whose mothers show high levels of depressive symptoms. First, to understand the impact that depressed parenting behavior may have on infants, researchers asked mothers to simulate depression while interacting with their infants (Cohn \& Tronick, 1983; Field, 1984; Field et al., 1986). These researchers found that infants' responses to nondepressed mothers' simulated depression were similar to infants' responses to the still-face interaction. Infants attempted to elicit responses from mothers by smiling briefly, and they showed an increase in distress and looking away from mothers compared with their behaviors in normal interaction. When mothers with high levels of depressive symptoms were asked to simulate depression, however, their infants behaved much like they did when their mothers were instructed to interact normally (Field, 1984).

On the basis of this research, it has been assumed that infants who have experienced a history of inconsistent or unresponsive social interactions with parents, as might occur with parental depression, will fail to show the normative responses to the stillface interaction. They may make fewer attempts to elicit social interaction with their parents and may become less distressed at the lack of responsiveness because it is familiar.

These assumptions have not been examined longitudinally as the mother-infant relationship develops and as maternal depression remits with time. The current study included a group of nondepressed mothers and a group of mothers diagnosed with postpartum depression when their infants were 2 months old. When the initially depressed mothers were assessed again when their infants were 6 months old, approximately $80 \%$ of these mothers' episodes of depression had remitted (Campbell, Cohn, \& Meyers, 1995). As previously reported, the mothers in this study who remained depressed at 6 months postpartum and their infants were significantly less positive in normal play interactions than were the other mother-infant dyads (Campbell et al., 1995). In the current study, we were interested in whether chronic maternal depression during the postpartum period would be related to infants' responses to the still-face interaction. We were also able to examine whether developmental change in infant responses to the still-face interaction occurred differentially for infants depending on the course of maternal depression.

\section{Individual Differences}

Theories of temperament propose that there are innate individual differences in positive and negative affectivity (e.g., Goldsmith, 1993; Kagan, Snidman, Arcus, \& Reznick, 1994). The still-face interaction presents infants with a mildly stressful social situation and is assumed to heighten individual differences, especially in the tendency to become distressed (Tronick et al., 1978). Little empirical work has examined the stability of individual differences in infant affective responses to the still-face interaction.

Rates of positive and negative responses that infants express during the still-face interaction show test-retest stability across a 2-week period (Tronick \& Weinberg, 1990) and between mother and father social partners (Braungart-Rieker, Garwood, Powers, \& Notaro, 1998). Studies of infant responses between 3 and 6 months are inconsistent. Two longitudinal studies have reported stability in self-regulatory behaviors (e.g., gazing away from mother and directed hand movements; Shapiro et al., 1998; Toda \& Fogel, 1993). One found significant stability in positive, but not in negative, responses from 3 to 6 months (Shapiro et al., 1998). In that study, anger and sadness expressions were analyzed separately, with stability coefficients of .27 and .22 , respectively. Because anger and sadness expressions are difficult to distinguish in infancy (Camras, 1992), it is possible that combining the two expressions in this study would have resulted in greater stability in the rate of negative responses. Toda and Fogel (1993) found instability in both positive and negative responses. In both studies, small sample sizes may have attenuated stability coefficients. In the current study, we examined stability in the rates of positive and negative responses and of gazing away from mother between 2,4 , and 6 months in a much larger sample of infants than has been studied previously.

\section{Affective Responses to the Still-Face Interaction as Predictors of Later Development}

Infancy is thought to be an important period for the emergence of characteristic affective styles (Calkins, 1994; Malatesta, Culver, Rich-Tesman, \& Shepard, 1989; Tronick, 1989). To the extent that responses to the still-face interaction reflect emerging affective styles, these behaviors may be markers of individual differences that persist beyond early infancy. In particular, infants who do not show normative responses to the still-face interaction may differ in important ways from other infants in terms of their characteristic affective styles and their subsequent social-emotional competence.

Very little research has examined infants' affective responses to the still-face interaction in relation to later affective behaviors. One study reported significant stability $(r=.57)$ between rates of infant distress responses to the still-face interaction at 6 months and distress responses in the separation episodes of the Strange Situation procedure at 15 months (Weinberg \& Tronick, 1991). With the exception of this study, we know of no research that has reported relations between responses to the still-face interaction during early infancy and later behaviors that reflect traitlike affective styles. Some prior research has found relations between infant 
responses to the still-face interaction at 6 months and later infant attachment classification (e.g., Bates, Maslin, \& Frankel, 1985; Cohn, Campbell, \& Ross, 1991; Kiser, Bates, Maslin, \& Bayles, 1986; Tronick, Ricks, \& Cohn, 1982). However, attachment theorists have argued strongly that infant attachment classifications are not based on traitlike affective styles (e.g., Sroufe, 1985).

The use of infant affective responses to the still-face interaction to assess the development of social-emotional competence and characteristic affective styles is a promising line of research (Tronick, 1989). In the current study, we assessed social-emotional competence at age 18 months by asking mothers to rate their children's typical problem behaviors that reflected externalizing and internalizing types of behavior. To understand the development of characteristic affective styles, we examined longitudinal relations between infant responses to the mother's still face at 6 months and 18-month social-emotional behaviors.

\section{Method}

\section{Participants}

Participants were 129 infants selected from a community sample of middle-class families with firstborn infants (for details of recruitment see Campbell, Cohn, Flanagan, Popper, \& Meyers, 1992) who completed at least one of the still-face interaction assessments at 2, 4, and 6 months. Mothers were all European American, married, and an average of 29 years old; all had at least a high school education, and $51 \%$ were college graduates. Infants were healthy, full-term singletons, 63 girls and 66 boys.

At the infants' ages of 2,4 , and 6 months, mothers were visited in their homes and interviewed with the Schedule for Affective Disorders and Schizophrenia (SADS; Spitzer, Endicott, \& Robins, 1978) modified for a postpartum sample (O'Hara, Neunaber, \& Zeboski, 1984). We rated mood and other symptoms of depression on a 6-point scale ranging from 1 (not present or present to a normal degree) to 6 (serious and incapacitating), following previous research (O'Hara et al., 1984). Diagnoses were assigned according to Research Diagnostic Criteria for major depressive disorder (Spitzer et al., 1978) on the basis of information about symptoms and symptom severity. Interviews were audiotaped for assessment of interrater reliability. Agreement on diagnoses, based on 39 cases, was $97 \%$. Interrater reliability on total symptom scores was .84. Additional information about assessment of depression, diagnostic interviews, and interrater agreement on diagnosis has been provided in previous reports (Campbell et al., 1992, 1995).

At study entry (infant age $=2$ months), 67 mothers met criteria for major depressive disorder. Depressed and nondepressed groups of women did not differ in age $(M \mathrm{~s}=29.7$ and 28.7 years, respectively) or in educational $(M \mathrm{~s}=5.7$ and 5.8) or occupational $(M \mathrm{~s}=5.5$ and 6.2$)$ levels as scored on the Hollingshead (1975) scale. Of the initially depressed mothers who were assessed again when their infants were 6 months old, 18 had episodes of depression that had remitted, 27 continued to show subclinical levels of depressive symptoms, and 19 continued to meet diagnostic criteria for major depressive disorder.

\section{Procedure}

Mothers and infants were visited in their homes at 2, 4, and 6 months, where they were videorecorded in a series of interactions, including feeding, face-to-face play, and still-face interactions. For these interactions, each mother was asked to choose a comfortable location in her home and to hold her infant on her lap or place her infant in an infant seat, whichever she preferred. In contrast to laboratory assessments, this method allowed us to observe mothers interacting with their infants in normal and familiar settings. Results involving the feeding and face-to-face play interactions were reported earlier (Campbell et al., 1995). The current article focuses on results from the still-face interaction, which differs in substantial ways from typical social interactions experienced by infants.

Still-face interactions. Prior to videorecording, mothers were given instructions for both the face-to-face play and still-face interactions. In the face-to-face play interactions, mothers were instructed simply to play with their babies as they normally would. Immediately following the 3-min face-to-face play interaction, mothers were told to turn away from their infants for $15 \mathrm{~s}$ and then to turn back toward their infants. Mothers were instructed to look at their infants for 3 min without responding in any way with facial or vocal expressions and were assured that the examiner would stop the still-face interaction if their infants became too distressed.

Data from the still-face interaction were available for 113 infants at 2 months, 123 infants at 4 months, and 116 infants at 6 months. Data were missing at the various ages because of mothers' inability to maintain the still face (described below), mothers' declining to participate in the home visit, technical difficulties, or babies' being too fussy or too sleepy to begin the interaction. Data at all three age points were available for 99 infants.

We videorecorded the interactions using a split-screen procedure so that the behaviors of both mothers and infants could be observed. Two cameras were used, with one focused on the infant and the other on the mother. The video output from the two cameras was combined with the use of a split-screen generator, and a digital time code was added to the videotape.

Assessment of mothers' behaviors. Prior to coding infant affective behaviors, we examined the videorecordings to determine if mothers adhered to the still-face instructions. In three interactions, mothers smiled briefly ( $3-5 \mathrm{~s})$, and infant data during these lapses were omitted from the analyses. Two or three mothers at each infant age were unable to maintain the still face (e.g., they smiled frequently, laughed, or talked). These interactions were deleted entirely from the data set. Approximately half of the mothers chose to hold their infants on their laps, and the other mothers chose to place their infants in seats. To assess the impact that holding may have had on infants' responses to the still-face interaction, we compared the affective responses of infants that were held with the affective responses of those seated in infant seats. We found no differences in their responses. This finding is consistent with prior research showing that active touching (e.g., rubbing the infant's arm, moving the infant's legs up and down playfully) changes infant responses to the still-face interaction (Pelaez-Nogueras, Field, Hossain, \& Pickens, 1996; Stack \& Muir, 1992) but that passive touching does not (Stack \& Muir, 1992).

Coding infant behaviors. Infants' behaviors were coded separately by trained coders unaware of mothers' diagnoses of depression and of the hypotheses about stability and change. In separate passes through the videotape, facial affect, direction of gaze, and type of vocalization were coded at 1 -s time intervals. In the current study, to maintain consistency with prior research, we coded facial affect as positive, neutral, or negative on the basis of behavioral descriptors specified in the Monadic Phases coding system (Tronick, Als, \& Brazelton, 1980). Gaze was coded as away from or toward the mother. Type of vocalization was coded as fuss/cry, coo/babble, or laugh. If the infant's face could not be seen (e.g., the infant turned away from the camera or the mother's face obscured the view of the infant's face), affect was coded as missing. Coding was conducted in real time whenever possible. If necessary, coders stopped the tapes and used slow-motion viewing to accurately identify changes in behavior. The same system was used to code infants' affect during the face-to-face play interactions with mothers (see Cohn, Campbell, Matias, \& Hopkins, 1990, for more information about procedures and methods used in the face-toface play interactions).

Reliability. To assess interobserver agreement, $20 \%$ of the interactions were selected randomly and coded by a second coder. Agreement was defined as both coders observing the same behavior within $\mathbf{l} \mathrm{s}$ of each other and was quantified with the use of percentage agreement and kappa to correct for chance agreement. Coders agreed on positive ( $97 \%$ agreement) and negative ( $82 \%$ agreement) expressions, which are the focus of the 
current study. Overall, coders reliably distinguished affective expressions (96\% agreement, $\kappa=.85$ ), direction of gaze (87\% agreement, $\kappa=.75$ ), and type of vocalization (93\% agreement, $\kappa=.82$ ).

Continuous measures of infant affective responses. To determine the amount of time during the interaction that an infant spent in positive states, negative states, and gazing away from the mother, we aggregated the time series of the two affect codes and gaze codes separately. As in prior research, neutral affect was not included in the analyses. Because the lengths of interactions varied (some were shortened if the infant became distressed, and amounts of missing data varied), the aggregate scores were expressed as a percentage of time. Thus, three continuous dependent variables were computed at each age, indicating the percentages of time during the interaction that the infant spent in positive and negative affective states and the percentage of time the infant spent gazing away from the mother. These measures are comparable to those studied in earlier examinations of developmental change and individual differences in infant affective responses to the still-face interaction (Gusella et al., 1988; Shapiro et al., 1998; Toda \& Fogel, 1993).

Categorizing infant affective responses. Prior research examining infant responses to the still-face interaction and later attachment relationships reported predictive relations between such relationships and the presence or absence of infant smiling or crying in response to the mother's still face (Cohn et al., 1991; Tronick et al., 1982). Because we planned to look at predictive relations between infant responses to the still-face interaction and later social-emotional behaviors, following this prior work, we created two dichotomous variables at each age. First, we categorized the continuous measure of positive affect into "presence of smiling" if the percentage of positive affect was greater than zero and "absence of smiling" otherwise. Second, we categorized the continuous measure of negative affect into "presence of crying" if negative affect occurred along with a vocal expression of fussing or crying at any time during the interaction and "absence of crying" otherwise. Gazing away was considered only as a continuous variable because an absence of gazing away never occurred. Thus, in addition to the continuous measures of percentages of positive and negative affect and of gazing away, two dichotomous dependent variables were created at each age that indicated the presence or absence of smiling and crying.

Maternal ratings of toddler problem behaviors. At 18 months, mothers $(n=88)$ rated the frequency (never, rarely, sometimes, or frequently) with which their children showed typical toddler problem behaviors using the Toddler Behavior Checklist (TBC; Larzelere, Martin, \& Amberson, 1989). The TBC was constructed as a downward extension of the widely used Child Behavior Checklist (CBCL; Achenbach, 1991, 1992) to assess the social-emotional development of young children from 9 months to 4 years of age. The CBCL provides a number of narrowband scales, along with Internalizing and Externalizing broadband scales, and a total problem behavior score. The TBC provides the following developmentally comparable narrowband scales, based on factor analyses of the 103 items on the checklist (Larzelere et al., 1989): Oppositional, Immaturity, Emotional Instability, Physical Aggression, and Shyness. Normative means on these scales for children ages 15-18 months (Korwin, 1992; Larzelere et al., 1989) and means for the current sample are as follows: Oppositional, normative sample $=33.70 /$ current sample $=34.53$; Immaturity, $19.00 /$ 16.03; Emotional Instability, 9.60/7.38; Physical Aggression, 17.20/15.00; and Shyness, 7.10/7.16.

To provide conceptual comparability to other widely used measures of child behavior problems, such as the CBCL (Achenbach, 1991, 1992), we computed an internalizing behavior score, an externalizing behavior score, and a total problem behaviors score. To compute the internalizing score, we summed the Immaturity, Emotional Instability, and Shyness scales $(\alpha=.82)$. Representative items that contributed to the internalizing score were "bites fingernails," "cries for no reason," "acts shy or timid," "clings to adults," "acts withdrawn," and "acts fearful or anxious." To compute the externalizing score, we summed the Oppositional and Physical Aggression scales $(\alpha=.91)$. Representative items that contributed to the externalizing score were "yells," "Ioses temper," "misbehaves after being told no," "uncooperative," and "hits others." The total problem behaviors score was the sum of the internalizing and externalizing scores.

Infants who were rated higher in internalizing behaviors were also rated higher in externalizing behaviors, $r(88)=.76, p<.001$, which is consistent with the high correlation (approximately .70) found between the Internalizing and Externalizing factors in normative samples (Achenbach, 1991; Achenbach, Edelbrock, \& Howell, 1987).

\section{Results}

\section{Preliminary Analyses}

In preliminary analyses of the study's dependent variables, we found that mother's socioeconomic status, which was based on educational and occupational levels, was uncorrelated with the percentages of infant positive affect, negative affect, and gazing away or with 18-month behavior problems ( $r$ s ranged from -.19 to .18 , all $n s$ ). Analyses of variance revealed no mean differences between male and female infants in percentages of positive affect, negative affect, and gazing away or in 18-month behavior problems.

Confirming expectations that infants would be sensitive to the change in mothers' social behavior between the normal face-toface play interactions and the still-face interactions, infants were significantly less positive, $F(1,93)=48.84, p<.001, \eta^{2}=.34$, and more negative, $F(1,93)=11.72, p<.01, \eta^{2}=.11$, in the still-face interaction than in the preceding normal face-to-face interaction with their mothers (for more information on motherinfant affective behavior during the normal face-to-face play interactions, see Campbell et al., 1995). Infants showed stable individual differences in positive and negative affect between the two interactions at each age (correlations ranged from .21 to .51 , all $p s<.05$ ). Mothers' positive affect and negative affect during the normal face-to-face interactions were unrelated to infant affect during the still-face interaction at all ages ( $r$ s ranged from -.12 to .18 , all $n s)$.

Preliminary analyses regarding 18-month behavior problems showed that mothers' and infants' positive affect and negative affect during normal face-to-face play interactions were uncorrelated with 18-month behavior problems ( $r$ s ranged from -.15 to .14 , all $n s$ ). To assess change in infant behavior between the normal and still-face interactions in relation to developmental outcomes, we computed change scores in infant affect between the two interactions at each age (for positive affect, $M$ s ranged from .09 at 2 months to .26 at 6 months; for negative affect, $M$ s ranged from .16 at 2 months to .19 at 6 months). The amount of change an infant showed at all ages in the percentages of positive and negative affect between the normal face-to-face play interactions and the still-face interactions was unrelated to 18 -month behavior problems ( $r$ s ranged from -.17 to .11 , all $n s$ ).

\section{Missing-Values Analysis}

Theories of infant emotion regulation propose that infants use gazing away to regulate negative affect. During the still-face interaction, infants normally become distressed and spend a large percentage of time gazing away from their mothers. If infants regularly turn away to the extent that coders cannot see their faces, 
it is possible that data in the still-face interaction are not missing at random. Through missing-values analysis, we found that the amount of missing data in the still-face interaction increased from 2 to 6 months, $F(2,196)=10.36, p<.001$, along with the percentages of gazing away. These findings suggest that the percentages of negative affect in the still-face interaction may be underestimated. Alternatively, even when their faces are not visible, infants may not be exhibiting negative affect because they are able to use gazing away from their mothers to regulate their distress. Furthermore, and consistent with other research (Cohn \& Tronick, 1987), infant gazing away also increased in normal faceto-face play interactions from 2 to 6 months, $F(2,214)=30.31$, $p<.001$, along with the amount of missing data, $F(2$, $214)=13.12, p<.001$. In normal interaction, infants may gaze away more frequently by 6 months, not to regulate distress but because they are becoming increasingly oriented toward objects and other stimuli in the room.

It is difficult to quantify the impact of missing values on the percentages of infant positive and negative affect in the still-face interaction, but the issue of nonrandom missing data should be taken into consideration when interpreting the results that follow. The dichotomous variables indicating the presence or absence of smiling and crying at any time during the still-face interaction are probably not affected by missing data to the same degree as are the continuous variables.

\section{Developmental Change in Infant Responses to the Still-Face Interaction}

To examine developmental change in the percentages of infant positive and negative responses and gazing away from the mother in the still-face interaction, we conducted repeated measures analyses of variance with age as the within-subject factor. Although no main effect for infant sex was found in preliminary analyses, we included infant sex to assess a possible Age $\times$ Sex interaction. To assess whether developmental change occurred differentially depending on the course of maternal depression, we included chronicity of maternal depression (remitted, subclinical, chronic, or nondepressed control) as a between-groups factor and tested the significance of the interaction between age and chronicity. Including chronicity of maternal depression as a between-groups factor also provided a test of the main effect of chronicity of maternal depression on infant responses to the still-face interaction. Results for the test of main effects of maternal depression are discussed in the next section.
Consistent with the results of other studies, no age-related developmental changes were found in the percentages of positive and negative responses to the mother's still face (see Table 1). Gazing away from the mother increased significantly from 2 to 6 months, $F(6,180)=25.17, p<.001$ (see Table 1). No significant interaction effects were found.

Developmental change in the presence or absence of expected responses (smiling and crying) between 2 and 4 months, between 4 and 6 months, and between 2 and 6 months was examined with the use of $2 \times 2$ chi-square analyses. Smiling and crying were the normative responses at each age. Smiling was observed in $64 \%$ of infants at 2 months, $69 \%$ at 4 months, and $68 \%$ at 6 months. No significant increase was found in the numbers of infants who smiled between 2 and 4 months, between 4 and 6 months, or between 2 and 6 months. Crying occurred for $52 \%$ of infants at 2 months, $59 \%$ at 4 months, and $67 \%$ at 6 months. No significant increase was found in the numbers of infants who cried between 2 and 4 months, between 4 and 6 months, or between 2 and 6 months. At each age, about one third of the infants both smiled and cried, about one third only smiled, and about one third cried but did not smile. Very few infants at each age did neither (12\% at 2 months, $8 \%$ at 4 months, and $4 \%$ at 6 months).

\section{Effects of Maternal Depression on Infant Responses to the Still-Face Interaction}

As described above, to assess the main effects of the chronicity of maternal depression on the percentages of infant positive and negative responses and gazing away from the mother, we conducted repeated measures analyses of variance with age as the within-subject factor and maternal chronicity of depression (remitted, subclinical, chronic, or nondepressed control) as the betweengroups factor. There was no effect for chronicity of maternal depression. In addition, levels of maternal depressive symptoms (total symptom scores) at each age were uncorrelated with percentages of infant positive affect $(r$ ranged from -.18 to -.03 , all $n s$ ) and negative affect ( $r$ s ranged from -.09 to .08 , all $n s$ ). Levels of maternal depressive symptoms were uncorrelated with infant gazing away at 2 months and at 6 months $(r s=.13$ and $.08, n s)$ but were correlated with gazing away at 4 months $(r=-.18, p<.05)$.

In terms of the presence or absence of expected responses, it was very unusual for an infant not to smile at any of the three age assessments $(n=7)$, but 4 of those 7 infants who did not were infants of mothers who were chronically depressed, $\chi^{2}(3, N=$ 126) $=10.15, p<.05$. At 6 months of age, there was a trend,

Table 1

Mean Percentages of Infant Affective Responses to Mother's Still Face

\begin{tabular}{|c|c|c|c|c|c|c|c|c|}
\hline \multirow[b]{2}{*}{ Affective response } & \multicolumn{2}{|c|}{2 months } & \multicolumn{2}{|c|}{4 months } & \multicolumn{2}{|c|}{6 months } & \multirow[b]{2}{*}{$F(6,190)$} & \multirow[b]{2}{*}{$\eta^{2}$} \\
\hline & $M$ & $S D$ & $M$ & $S D$ & $M$ & $S D$ & & \\
\hline Positive affect & .06 & .09 & .07 & 10 & .07 & .09 & .16 & \\
\hline Negative affect & .26 & .33 & .22 & .28 & .26 & .31 & .98 & \\
\hline Gazing away & .51 & .37 & .73 & .24 & .79 & .21 & $25.17 * * *$ & .24 \\
\hline
\end{tabular}

Note. $\eta^{2}=$ effect size.

$* * * p<.001$. 
although not a statistically significant one, for infants of mothers who were chronically depressed to be less likely to smile than infants of mothers who no longer met criteria for clinical depression, $\chi^{2}(2, N=59)=5.88, p=.053$. Chronicity of maternal depression was unrelated to the presence or absence of infant crying in the still-face interactions.

\section{Individual Differences in Infant Affective Responses to the Still-Face Interaction}

The percentage of negative affect in the still-face interaction was unstable from 2 to 4 months but showed modest stability from 4 to 6 months, $r(113)=.30, p<.001, r^{2}=.09$. The percentage of positive affect was unstable from 2 to 6 months. There were stable individual differences in self-regulation of distress, as indicated by stability in gazing away from the mother from 2 to 4 months, $r(108)=.26, p<.01, r^{2}=.07$, and from 4 to 6 months, $r(113)=.26, p<.01, r^{2}=.07$. In terms of the presence or absence of expected responses, chi-square analyses indicated that infants who smiled at earlier ages in response to the still-face interaction were not more likely than other infants to smile at subsequent ages. Similarly, infants who cried at earlier ages were not more likely to cry at later ages.

\section{Amounts of Infant Affective Responses to the Still-Face Interaction as Predictors of Later Development}

The percentages of infant positive and negative affect and gazing away in response to the mother's still face at all ages were unrelated to 18-month total behavior problems, internalizing behaviors, or externalizing behaviors ( $r$ s ranged from -.18 to .01 , all $n s$ ). To follow up on these correlational analyses, we ranked infants into terciles indicating high, moderate, and low percentages of positive affect, terciles indicating high, moderate, and low percentages of negative affect, and terciles indicating high, moderate, and low percentages of gazing away. Rankings were computed separately at each age.

Infants who expressed high, moderate, and low percentages of positive affect at 6 months did not differ in 18-month total problem behaviors or in 18-month internalizing behaviors. They did differ in 18-month externalizing behaviors, $F(2,78)=3.35, p<.05$, $\eta^{2}=.04$. Infants who were the least positive in the still-face interaction at 6 months, relative to other infants, were rated by their mothers as showing higher levels of externalizing behaviors at 18 months $(M s=53.32$ for infants with low percentages of positive affect, 44.00 for those with moderate percentages, and 48.02 for those with high percentages; Student NewmanKeuls post hoc test, $p<.05$ ).

Infants who expressed high, moderate, and low percentages of negative affect at 6 months differed in 18-month total problem behaviors, $F(2,78)=3.81, p<.05, \eta^{2}=.09$, and in 18 -month internalizing behaviors, $F(2,78)=6.31, p<.05, \eta^{2}=.14$, but not in 18-month externalizing behaviors. Infants who were moderately negative in the still-face interaction at 6 months, relative to other infants, were rated by their mothers as showing higher levels of total problem behaviors $(M s=72.44,87.65$, and 76.21 for infants with low, moderate, and high percentages of negative affect, respectively; Student-Newman-Keuls post hoc test, $p<.05$ ) and higher levels of internalizing behaviors $(M \mathrm{~s}=26.04,34.76$, and 30.33 for infants with low, moderate, and high percentages of negative affect, respectively; Student-Newman-Keuls post hoc test, $p<.05$ ). Infants who expressed high, moderate, and low percentages of gazing away from their mothers at 6 months did not differ significantly in total problem behaviors, internalizing behaviors, or externalizing behaviors at 18 months.

\section{Presence or Absence of Infant Affective Responses to the Still-Face Interaction as a Predictor of Later Development}

The presence or absence of expected responses (smiling and crying) to the still-face interaction at 6 months more clearly predicted 18-month internalizing and externalizing behaviors, although not total problem behaviors. There was a trend for infants who did not smile in the still-face interaction at 6 months to have greater levels of total problem behaviors at 18 months, $F(1$, 79) $=3.82, p=.054$, although this trend was not statistically significant. Similar results were found for the presence or absence of crying in predicting total problem behaviors, $F(1,79)=3.93$, $p=.051$.

At 18 months, infants who did not cry at all in response to the still-face interaction at 6 months were rated as showing fewer internalizing behaviors, such as being shy, withdrawn, or moody, than were other toddlers, $F(1,79)=6.14, p<.05, \eta^{2}=.06$ (see Table 2). Infants who did not smile at all in the still-face interaction at 6 months, compared with those who did, were rated by their mothers at 18 months as showing more externalizing behaviors, including opposition and aggression, $F(1,79)=5.44, p<.05$, $\eta^{2}=.07$ (see Table 2). With one exception, predictive relations occurred only for infant responses to the still-face interaction at 6 months. Infants who did not cry at both 4 and 6 months were rated by their mothers as showing fewer internalizing behaviors at 18 months, $F(1,77)=5.41, p<.05, \eta^{2}=.07$. There were no differences in problem behaviors among infants who cried only, infants who smiled only, and infants who did both at 6 months.

The chronicity of maternal depression was unrelated to 18 month problem behaviors, although mothers' reports of infant problem behaviors at 18 months were correlated with current levels of maternal depressive symptoms. Mothers' levels of depressive symptoms were correlated with reports of 18 -month internalizing behaviors $\left(r=.22, p<.05, r^{2}=.05\right)$ and externalizing behaviors $\left(r=.31, p<.05, r^{2}=.10\right)$.

To examine the relative contributions of mothers' current levels of depressive symptoms and the presence or absence of expected affective responses to the still-face interaction at 6 months as

Table 2

Mean Levels of Infant Problem Behaviors at 18 Months by Presence or Absence of Smiling and Crying in Response to Mother's Still Face at 6 Months

\begin{tabular}{lccccc}
\hline & \multicolumn{2}{c}{ Cried } & & \multicolumn{2}{c}{ Smiled } \\
\cline { 2 - 3 } \cline { 6 - 7 } Problem behavior & Yes & No & & Yes & No \\
\hline Internalizing & $31.75_{\mathrm{a}}$ & $26.28_{\mathrm{b}}$ & & 29.20 & 31.68 \\
Externalizing & 50.06 & 45.52 & & $46.20_{\mathrm{a}}$ & $53.32_{\mathrm{b}}$ \\
\hline
\end{tabular}

Note. Means within rows that do not share subscripts differ at $p<.05$. 
predictors of 18-month problem behaviors, we conducted separate regression analyses for internalizing and externalizing behaviors. Mothers' current levels of depressive symptoms explained about $5 \%$ of the variation in their reports of infant internalizing behaviors at 18 months, $R^{2}=.048, F(1,79)=4.03, p<.05, \beta=.23$. Whether an infant cried in response to the still-face interaction at 6 months explained about $8 \%$ of the variation in internalizing behaviors, change in $R^{2}=.077, F(2,78)=6.87, p<.05, \beta=.28$. The order of entry did not change the results, indicating that these variables were independent sources of variation in 18-month internalizing behaviors.

For externalizing behaviors, mothers' current levels of depressive symptoms explained about $10 \%$ of the variation in reports of 18 -month behaviors, $R^{2}=.098, F(1,79)=8.64, p<.01, \beta=.31$. Whether an infant smiled in the still-face interaction at 6 months explained about $6 \%$ of the variation in externalizing behaviors, change in $R^{2}=.06, F(2,78)=5.58, p<.05, \beta=-.25$. Again, order of entry did not change the results, indicating that mothers' current depressive symptoms and infants' affective responses to the still-face interaction were independent influences on 18-month externalizing behavior problems.

\section{Discussion}

The still-face interaction, in which mothers or fathers are instructed to become unresponsive to their infants after a period of normal social play, generates predictable responses from infants. An infant may smile briefly at his or her parent to try to regain attention, become distressed at the lack of responsiveness, even to the point of crying, or look away to regulate the distress he or she may be feeling. In the current study, we observed infants' responses to the still-face interaction at 2, 4, and 6 months. Our findings showed that the normative responses described above occur at an earlier age than previously studied and suggest that infants are sensitive to disruptions in social reciprocity as early as 2 months of age.

Social-emotional development undergoes rapid change between 2 and 6 months of age (Cohn \& Tronick, 1987, 1988; Kaye $\&$ Fogel, 1980). Given that infants' expressions of positive emotion and responsiveness during normal play interactions increase during this period (Cohn \& Tronick, 1987; Kaye \& Fogel, 1980), we expected that there would be age-related changes in rates of infants' positive and negative responses to the still-face interaction as well. Presumably, infants' increased experience with and expectations of social responsiveness from 2 to 6 months would increase their sensitivity to the still-face interaction. However, consistent with the results of previous studies, we found no increase in infant positive and negative affective responses.

One methodological explanation for this negative finding may be the possibility of over- or underestimates of rates of negative and positive affective responses at the various ages because of missing data. When infants turned away from the camera, there were intervals of missing values for measures of positive and affective responses, although not for measures of gazing away. Because it is assumed that infants use gazing away to regulate emotion, we may be losing information about the valence of infants' affective responses during the times they turn far enough away that their faces cannot be seen. This problem does not have an easy solution, but it should be considered in subsequent re- search on infant affective responses to the still-face interaction. Note that this issue does not have an impact on the assessment of gazing away, for which we did find an increase from 2 to 6 months.

A substantive explanation for the lack of observed developmental change in rates of positive and negative responses is that as infants become self-sufficient in regulating their emotion responses, they are able to manage increased distress more effectively and have less need to elicit assistance from their parents by crying or smiling. This explanation is supported by the finding that the only response to show significant change from 2 to 6 months was an increase in gazing away from the mother, an important emotion-regulation strategy. If this explanation is accurate, the structural organization of infants' positive, negative, and emotionregulating (e.g., gazing away) responses should change over time. The structural organization of infants' responses refers to predictable and meaningful sequences of behaviors. For example, Cohn and Tronick (1983) used lag-sequential analysis to compare the responses of 6-month-old infants to their mothers behaving normally with their responses to their mothers simulating depression. In normal social interactions, infants cycled between positive and neutral, attentive states. In the simulated depression condition, infants cycled between negative states and gazing away, as infants' need to regulate distress increased. Similar shifts may occur across development if infants' abilities to self-regulate emotion increase along with distress from their mothers' unresponsiveness. We have planned future research to examine sequences of infants' positive, negative, and emotion-regulation responses to the still-face interaction across development.

Stable individual differences in the tendency to become distressed by the mother's unresponsiveness and in the use of gazing away to regulate emotion were evident across early infancy, consistent with theories of traitlike affective styles (Goldsmith, 1993; Kagan et al., 1994; Tronick, 1989). Although stability coefficients were low to moderate and effect sizes were relatively small, this stability is nonetheless impressive given the brief sampling of behaviors across 2-month intervals. Furthermore, the presence or absence of smiling and crying in the still-face interaction by 6 months of age predicted mothers' descriptions of their toddlers' affective behaviors at 18 months. Smiling to try to re-engage mothers' attention in the still-face interaction was a normative response at 6 months. Infants who failed to show this sociable behavior were described 1 year later as more aggressive and oppositional than were other children. Similarly, the presence of crying was a normative response to the still-face interaction at 6 months. Infants who failed to cry were rated by their mothers as showing fewer internalizing types of behaviors at 18 months, such as withdrawn behavior, shyness, and moodiness. These children were described as being more mature than other toddlers. Again, effect sizes were relatively small, but prediction from experimenters' observations of infant behaviors during a brief 3-min interaction to mothers' ratings of behavior 1 year later is striking. Also noteworthy is the theoretical consistency of prediction, with lack of smiling at 6 months predicting more aggressive and oppositional behaviors and lack of crying at 6 months predicting more mature behaviors.

It is important to note that the behaviors of all infants in the study were within the normal range of variation, and the results do not suggest that responses to the still-face interaction predict 
clinically significant behavior problems. It would be interesting to examine whether responses to the still-face interaction in early infancy predict affective styles a year later in infants with difficult temperaments or infants who have an older sibling identified with internalizing or externalizing behavior problems.

In the still-face interaction, very few infants never tried to elicit a response from their mothers with a smile at any of the three age assessments. Of note is that more than half of the infants who never smiled had mothers who were chronically depressed. The associations we found between lack of infant smiling in the stillface interaction and later externalizing behaviors suggest an interplay between maternal depression, diminished expressions of positive affect, and child externalizing behaviors. Prior research on the association between maternal depression and child externalizing behaviors and between parenting in general and child externalizing behaviors has focused on high rates of negative and controlling parenting behaviors. Our results suggest an important, and possibly independent, role for parental positive affect (or lack thereof) in the development of child externalizing behaviors.

We also found that current maternal depression and infants' earlier affective responses to the still-face interaction made independent but comparable contributions to the prediction of mothers' ratings of internalizing and externalizing behaviors at 18 months. Levels of depression at the time of mothers' ratings appeared to have a somewhat stronger role in the prediction of toddler externalizing behaviors than in the prediction of internalizing behaviors, which may reflect the salience of these types of behaviors for parents, particularly under higher levels of depressive symptoms. Together, our findings support current transactional models of child development that emphasize the contributions of both child and parent characteristics to the emergence of child behavior problems.

Nevertheless, in the current study, infants of chronically depressed mothers did not have higher levels of problem behaviors at 18 months. They also appeared to become as distressed as did other infants and to use gazing away to regulate emotion as effectively as did other infants. The mothers and infants in this study were from a demographically low-risk population, and the results might vary for other populations that are subject to additional stressors and lack of resources. The current results underscore the need to understand more clearly the various ways in which depression may affect parenting behavior and to recognize that other factors related to depression (e.g., financial stresses, lack of support) may account in part for the negative developmental outcomes often associated with children of parents with depression.

The results of the current study highlight the complex interplay between social and emotional development. Our findings lend support to theories that infants rapidly develop social competence and expectations of reciprocal social behavior. As early as 2 months of age, infants actively elicit interaction from their mothers when their mothers do not respond to them with facial expressions or their voices. By 6 months of age, most infants appear to have developed fairly effective strategies to manage distress when mothers do not respond to them immediately. Infant responses to the socially stressful still-face interaction may reflect temperamental differences in early childhood and emerging individual differences in social competence. Although subsequent experience is likely to influence the development of characteristic behavioral styles, our results suggest that infants who do not show the expected responses to the still-face interaction by 6 months may differ from other children in important ways of relating to the social world.

\section{References}

Achenbach, T. M. (1991). Manual for the Child Behavior Checklist/4-18 \& 1991 profile. Burlington, VT: University of Vermont, Department of Psychiatry.

Achenbach, T. M. (1992). Manual for the Child Behavior Checklist/2-3 and 1992 profile. Burlington, VT: University of Vermont, Department of Psychiatry.

Achenbach, T. M., Edelbrock, C., \& Howell, C. T. (1987). Empiricallybased assessment of the behavioral/emotional problems of 2-3 year-old children. Journal of Abnormal Child Psychology, 15, 629-650.

Bates, J. E., Maslin, C. A., \& Frankel, K. A. (1985). Attachment security, mother-child interaction, and temperament as predictors of behaviorproblem ratings at age three years. Monographs of the Society for Research in Child Development, 50, 167-193.

Braungart-Rieker, J., Garwood, M. M., Powers, B. P., \& Notaro, P. C. (1998). Infant affect and affect-regulation during the still-face paradigm with mothers and fathers: The role of infant characteristics and parental sensitivity. Developmental Psychology, 34, 1428-1437.

Calkins, S. D. (1994). Origins and outcomes of individual differences in emotion regulation. In N. A. Fox (Ed.), The development of emotion regulation, Monographs of the Society for Research in Child Development, 59(2-3, Serial No. 240).

Campbell, S. B., Cohn, J. F., \& Flanagan, C., Popper, S., \& Meyers, T. (1992). Course and correlates of postpartum depression during the transition to parenthood. Development and Psychopathology, 4, 29-47.

Campbell, S. B., Cohn, J. F., \& Meyers, T. (1995). Depression in first-time mothers: Mother-infant interaction and depression chronicity. Developmental Psychology, 60, 349-357.

Camras, L. A. (1992). Expressive development and basic emotions. Cognition and Emotion, 6, 269-283.

Cohn, J. F., Campbell, S. B., Matias, R., \& Hopkins, J. (1990). Face-to-face interactions of postpartum depressed and nondepressed mother-infant pairs at 2 months. Developmental Psychology, 26, 15-23.

Cohn, J. F., Campbell, S. B., \& Ross, S. (1991). Infant response in the still-face paradigm at 6 months predicts avoidant and secure attachment at 12 months. Development and Psychopathology, 3, 367-376.

Cohn, J. F., \& Tronick, E. Z. (1983). Three-month-old infants' reaction to simulated maternal depression. Child Development, 54, 185-193.

Cohn, J. F., \& Tronick, E. Z. (1987). Mother-infant interaction: The sequence of dyadic states at 3,6, and 9 months. Developmental Psychology, 23, 68-77.

Cohn, J. F., \& Tronick, E. Z. (1988). Mother-infant interaction: The influence is bidirectional and unrelated to periodic cycles in either partner's behavior. Developmental Psychology, 24, 386-392.

Field, T. (1984). Early interactions between infants and their postpartum depressed mothers. Infant Behavior and Development, 7, 517-522.

Field, T., Vega-Lahr, N., Scafidi, F., \& Goldstein, S. (1986). Effects of maternal unavailability on mother-infant interactions. Infant Behavior and Development, 9, 473-478.

Goldsmith, H. H. (1993). Temperament: Variability in developing emotion systems. In M. Lewis \& J. M. Haviland (Eds.), Handbook of emotions (pp. 353-364). New York: Guilford Press.

Gusella, J., Muir, D., \& Tronick, E. (1988). The effect of manipulating maternal behavior during an interaction on three- and six-month-olds' affect and attention. Child Development, 59, 1111-1124.

Hollingshead, A. (1975). Four-factor index of social status. Unpublished manuscript, Yale University.

Kagan, J., Snidman, N., Arcus, D., \& Reznick, S. J. (1994). Galen's prophecy: Temperament in human nature. New York: Basic Books. 
Kaye, K., \& Fogel, A. (1980). The temporal structure of normal communication between mothers and infants. Developmental Psychology, 16, $454-464$.

Kiser, L. J., Bates, J. E., Maslin, C. A., \& Bayles, K. (1986). Mother-infant play at 6 months as a predictor of attachment security at 13 months. Journal of the American Academy of Child Psychiatry, 25, 68-75.

Korwin, L. L. (1992). Utility of the Toddler Behavior Checklist in identifying a clinical sample. Unpublished doctoral dissertation, University of Hartford, Hartford, CT.

Larzelere, R. E., Martin, J. A., \& Amberson, T. G. (1989). The Toddler Behavior Checklist: A parent-completed assessment of social-emotional characteristics of young preschoolers. Family Relations, 38, 418-425.

Malatesta, C. Z., Culver, C., Rich-Tesman, J., \& Shepard, B. (1989). The development of emotion expression during the first two years of life. Monographs of the Society for Research in Child Development, 54 (1-2, Serial No. 219).

Murray, L., \& Trevarthen, C. (1985). Emotional regulation of interactions between two-month-olds and their mothers. In T. Field \& N. Fox (Eds.), Social perception in infants (pp. 177-197). Norwood, NJ: Ablex.

O'Hara, M. W., Neunaber, D. J., \& Zeboski, E. M. (1984). Prospective study of postpartum depression: Prevalence, course, and predictive factors. Journal of Abnormal Psychology, 93, 158-171.

Pelaez-Nogueras, M., Field, T. M., Hossain, Z., \& Pickens, J. (1996). Depressed mothers' touching increases infants' positive affect and attention in still-face interactions. Child Development, 67, 1780-1792.

Shapiro, B., Fagen, J., Prigot, J., Carroll, M., \& Shalan, J. (1998). Infants' emotional and regulatory behaviors in response to violations of expectancies. Infant Behavior \& Development, 27, 299-313.

Spitzer, R. S., Endicott, J., \& Robins, E. (1978). Research Diagnostic Criteria: Rationale and reliability. Archives of General Psychiatry, 36, $773-782$.

Sroufe, L. A. (1985). Attachment classification from the perspective of infant-caregiver relationships and infant temperament. Child Development, 56, 1-14.

Stack, D. M., \& Muir, D. W. (1992). Adult tactile stimulation during face-to-face interactions modulates five-month-olds' affect and attention. Child Development, 63, 1509-1525.

Toda, S., \& Fogel, A. (1993). Infant response to the still-face situation at 3 and 6 months. Developmental Psychology, 29, 532-538.

Tronick, E. Z. (1989). Emotions and emotional communication in infants. American Psychologist, 44, 112-119.

Tronick, E. Z., Als, H., Adamson, L., Wise, S., \& Brazelton, B. (1978). The infants' response to entrapment between contradictory messages in face-to-face interaction. American Academy of Child Psychiatry, 1 , $1-13$.

Tronick, E. Z., Als, H., \& Brazelton, T. B. (1980). Monadic phases: A structural descriptive analysis of infant-mother normal interaction. Merrill-Palmer Quarterly, 26, 3-24.

Tronick, E. Z., Ricks, M., \& Cohn, J. F. (1982). Maternal and infant affective exchanges: Patterns of adaptation. In T. Field \& A. Fogel (Eds.), Emotion and interaction: Normal and high risk infants (pp. 83-100). Hillsdale, NJ: Erlbaum.

Tronick, E. Z., \& Weinberg, M. K. (1990, April). Emotion regulation in infancy: Stability of regulatory behaviors. Paper presented at the International Conference on Infant Studies, Montreal, Quebec, Canada.

Weinberg, M. K., \& Tronick, E. Z. (1991, April). Stability of infant social and coping behaviors and affective displays between 6 and 15 months: Age-appropriate tasks and stress bring out stability. Paper presented at the Society for Research in Child Development, Seattle, WA.

Weinberg, K. M., \& Tronick, E. Z. (1994). Beyond the face: An empirical study of infant affective configurations of facial, vocal, gestural, and regulatory behaviors. Child Development, 65, 1503-1515.

Weinberg, K. M., \& Tronick, E. Z. (1996). Infants' affective reactions to the resumption of maternal interaction after the still-face. Child Development, 67, 905-914.
Received May 19, 2000

Revision received April 16, 2001

Accepted April 16, 2001 